\title{
EFETIVIDADE DO EXERCÍCIO PÉLVICO NO PERIOPERATÓRIO DE PROSTATECTOMIA RADICAL: REVISÃO DE LITERATURA
}

\author{
Effectiveness of pelvic floor exercises in the perioperative \\ period of radical prostatectomy: a literature review
}

\author{
Efectividad del ejerciciopara elsuelopélvicoen el perioperatorio \\ de prostatectomía radical: revisión de literatura
}

Artigo de Revisão

\section{RESUMO}

Objetivo: Realizar uma revisão na literatura sobre a efetividade dos exercícios pélvicos no perioperatório de prostatectomia radical. Métodos: A partir dos descritores em saúde (DeCS) - (prostatectomia) AND (fisioterapia) AND (assoalho pélvico) AND (incontinência urinária) -, foram selecionados artigos nas línguas inglesa, espanhola e portuguesa, independentemente do ano de publicação. Após a busca na base de dados da BVS, observaram-se 26 estudos: 17 da MEDLINE, 5 da IBECS, 2 da LILACS e 2 da CENTRAL. Desse total, 17 foram excluídos por não se adequarem aos critérios de inclusão da pesquisa, culminando em um total de 9 artigos, os quais foram analisados no presente estudo. Resultados: Os exercícios perioperatórios de fortalecimento da musculatura pélvica para prostatectomia apresentam resultados importantes quanto à minimização da incontinência urinária, tendo em vista o forte impacto do problema sobre a qualidade de vida dos pacientes. Conclusão: As evidências científicas apontam que, apesar da heterogeneidade das técnicas e amostras, os exercícios perioperatórios apresentam resultados promissores quanto à minimização da incontinência urinária pós-operatório, principalmente acelerando a recuperação e cura ou diminuindo os sintomas.

Descritores: Prostatectomia; Fisioterapia; Diafragma da Pelve; Incontinência Urinária.

\section{ABSTRACT}

Objective: To review the literature on the effectiveness of pelvic floor exercises in the perioperative period of radical prostatectomy. Methods: By using the health descriptors (DeCS) tw (prostatectomy) AND (physiotherapy) AND (pelvic floor) AND (urinary incontinence), articles were selected in English, Spanish and Portuguese, regardless of the year of publication. After searching in the BVS database, 26 studies were found: 17 in MEDLINE, 5 in IBECS, 2 in LILACS, and 2 in CENTRAL. Of this total, 17 were excluded because they did not meet the study inclusion criteria, culminating in a total of 9 articles, which were analyzed in this study. Results: Pelvic floor muscle strengthening exercises in the prostatectomy perioperative period have important results in terms of minimizing urinary incontinence, considering the strong impact of the problem on the patients' quality of life. Conclusion: Scientific evidence points out, despite the heterogeneity of techniques and samples, that the perioperative exercises show promising results in reducing postoperative urinary incontinence, mainly speeding recovery and healing or reducing the symptoms.

Descriptors: Prostatectomy; Physical Therapy Specialty; Pelvic Floor; Urinary Incontinence.

\author{
Adonivia Guimarães Santos ${ }^{(1)}$ \\ Nayara Alexandre de Souza de \\ Almeida ${ }^{(1)}$ \\ Luisa Braga Jorge ${ }^{(2)}$ \\ Stanley Soares Xavier ${ }^{(3)}$ \\ Gustavo Sutter Latorre ${ }^{(1)}$
}

1) Faculdade Inspirar - Curitiba (PR) Brasil

2) Pontifícia Universidade Católica PUCRS - Porto Alegre (RS) - Brasil

3) Escola Superior da Amazônia ASAMAZ - Belém (PA) - Brasil

Recebido em: 18/10/2015 Revisado em: 27/11/2015 Aceito em: 07/01/2016 


\section{RESUMEN}

Objetivo: Realizar una revisión de la literatura sobre la efectividad de los ejercicios para el suelo pélvico en el peri operatorio de prostatectomía radical. Métodos: Basado en los descriptores de salud (DeCS) - (prostatectomía) AND (fisioterapia) AND (suelo pélvico) AND (incontinencia urinaria) - fueron elegidos artículos en el idioma inglés, español y portugués independiente del año de publicación. Tras la búsqueda en la base de datos de la BVS se observaron 26 estudios: 17 de MEDLINE, 5 de la IBECS, 2 de LILACS y 2 de la CENTRAL. Del total, 17 fueron excluidos porque no correspondian a los criterios de inclusión de la investigación, finalizando un total de 9 artículos los cuales fueron analizados en este estudio. Resultados: Los ejercicios del perioperatorio para fortalecer la musculatura pélvica para la prostatectomía presentaron resultados importantes para la minimización de la incontinencia urinaria considerando el fuerte impacto del problema sobre la calidad de vida de los pacientes. Conclusión: Las evidencias cientificas muestran que a pesar de la heterogeneidad de las técnicas y muestras, los ejercicios en el perioperatorio presentan resultados positivos para la minimización de la incontinencia urinaria en el posoperatorio acelerando la recuperación y la cura o disminuyendo los sintomas.

Descriptores: Prostatectomía; Fisioterapia; Diafragma Pélvico; Incontinencia Urinaria.

\section{INTRODUÇÃO}

A Prostatectomia Radical (PR) é uma das principais modalidades de tratamento utilizada para ressecção de tumores da próstata, descrita como procedimento eficaz no tratamento primário do câncer de próstata $(\mathrm{CP})$ localizado $^{(1,2)}$. É particularmente indicada para pacientes com doença em estágios A e B - T1 e T2. Quando o tumor atinge os tecidos periprostáticos, ou seja, estágio $\mathrm{C}-\mathrm{T} 3 \mathrm{e}$ T4, a cirurgia não remove integramente a neoplasia e os pacientes são melhores tratados com radioterapia radical ${ }^{(3)}$.

Embora propicie elevadas taxas de cura, a cirurgia é frequentemente acompanhada de complicações, sendo a incontinência urinária (IU) e a disfunção erétil (DE) as mais importantes ${ }^{(4)}$. Atualmente, as técnicas cirúrgicas apresentam incidência de complicações reduzida. As mais utilizadas são a ressecção transuretral (RTU), PR aberta ou videolaparoscopia - esta última é a que apresenta menor tempo de internação, menor morbidade pós-operatória e menores custos ${ }^{(5)}$.

$\mathrm{Na} P R$, a uretra prostática é removida, sendo o controle urinário mantido através do colo da bexiga e esfíncter urinário externo. As lesões anatômicas intrínsecas ao procedimento tendem a tornar a junção uretrovesical menos favorável à manutenção da continência urinária, gerando uma maior pressão sobre o esfíncter uretral externo ${ }^{(6)} \mathrm{e}$ ocasionando o aparecimento da IU. Esta é definida como qualquer perda involuntária de urina, sendo caracterizada pelo impacto negativo nos âmbitos emocional, social e econômico do indivíduo e em seus amigos, familiares e cuidadores $^{(7)}$.

A PR está entre os principais procedimentos cirúrgicos causadores de $\mathrm{IU}^{(8,9)}$ - a incidência de IU após a PR varia entre $0,5 \%$ e $87 \%$ nos primeiros seis meses e de $5 \%$ a $44 \%$ no primeiro ano após a cirurgia ${ }^{(10-12)}$.

Tanto a função urinária quanto a erétil estão relacionadas à função da musculatura do assoalho pélvico (MAP), que deve contrair-se para manter a continência urinaria e relaxar para permitir os esvaziamentos intestinal e vesical, mantendo ainda a posição anatômica dos órgãos pélvicos ${ }^{(13)}$.

O treinamento funcional do assoalho pélvico é um método de contração específico, cujos exercícios focam na prevenção e no tratamento de todos os tipos de perturbações na região abdominal, pélvica e coluna lombar ${ }^{(14,15)}$.

A fisioterapia pélvica vem emergindo como tratamento potencial para as sequelas urinárias pós-PR, proporcionando melhoria na qualidade de vida e, consequentemente, na saúde $^{(16)}$. No entanto, ainda não está elucidada a efetividade desse tipo de tratamento sobre a IU pós-prostatectomia, sendo esse esclarecimento o objetivo principal do presente estudo.

\section{MÉTODOS}

Realizou-se um estudo do tipo revisão bibliográfica a partir de ensaios clínicos controlados nos quais as técnicas fisioterapêuticas de fortalecimento muscular foram utilizadas para o tratamento no período perioperatório de prostatectomia radical.

A busca dos estudos foi realizada na base de dados da Biblioteca Virtual em Saúde (BVS), a partir dos descritores em saúde (DeCS): (prostatectomia radical) AND (modalidades de fisioterapia) AND (instance: "regional")

Os critérios de elegibilidade do estudo foram artigos científicos publicados nas línguas inglesa, espanhola e portuguesa, independentemente do ano de publicação do manuscrito, haja vista a escassez de literatura acerca da temática.

Foram excluídos os artigos em duplicata e que não citassem a fisioterapia como tratamento principal na conduta dos estudos.

A seleção dos estudos ocorreu por meio da análise do título, resumo e texto completo, respectivamente. 
Quadro I - Resumo dos estudos utilizados na revisão.

\begin{tabular}{|c|c|c|c|}
\hline Autor/ano & Número de participantes & Procedimentos de intervenção & Principais resultados \\
\hline $\begin{array}{l}\text { Centemero et al., } \\
2010^{(17)}\end{array}$ & $\begin{array}{l}118 \\
\text { - Grupo A: Exercícios para a } \\
\text { musculatura do assoalho pélvico } \\
\text { no pré e pós-operatório em sua } \\
\text { residência }(\mathrm{n}=59) \text {. } \\
\text {-Grupo B: Exercícios para o } \\
\text { fortalecimento do assoalho pélvico } \\
\text { pós-operatório e na residência } \\
(\mathrm{n}=59) \text {. }\end{array}$ & $\begin{array}{l}\text { Grupo A: } \\
\text { Quatro semanas antes da cirurgia. (duas } \\
\text { vezes por semana, durante } 30 \text { minutos, } \\
\text { no ambulatório e na residência do } \\
\text { participante). } \\
\text { Grupo B: } \\
48 \text { horas depois da retirada do cateter. } \\
\text { Atividades realizadas: } \\
\text { Contração do assoalho pélvico. }\end{array}$ & $\begin{array}{l}\text { Grupo A: Atingiu } 44,1 \% \text { ( } 26 \text { de } 59) \\
\text { de continentes em seu grupo. } \\
\text { Grupo B: Conseguiu 20,3\% (12 de } \\
\text { 59) de continentes no grupo. }\end{array}$ \\
\hline $\begin{array}{l}\text { Geraerts } \\
\text { et al., } 2013^{(18)}\end{array}$ & $\begin{array}{l}180 \\
\text { - Grupo intervenção: Realizou um } \\
\text { programa de TMAP (desde o período } \\
\text { pré-operatório até o pós-operatório) } \\
\text { controlado pelo terapeuta e fornecido } \\
\text { com biofeedback eletromiográfico } \\
\text { (n=91). } \\
\text {-Grupo controle: Realizou o mesmo } \\
\text { tratamento do grupo intervenção, } \\
\text { porém, somente após a retirada do } \\
\text { cateter (pós-operatório) }(\mathrm{n}=89) \text {. }\end{array}$ & $\begin{array}{l}\text { Grupo experimental: } \\
\text { Começou a exercer } \\
3 \text { semanas antes da cirurgia. Os pacientes } \\
\text { receberam uma sessão de } 30 \text { min. de } \\
\text { TMAP por semana. Além disso, realizaram } \\
\text { um programa em casa de } 60 \text { contrações por } \\
\text { dia e foram instruídos sobre a contração } \\
\text { dos } \\
\text { músculos do assoalho pélvico ao tossir, } \\
\text { sentar-se ou levantar-se de uma cadeira. } \\
\text { Grupo controle: } \\
\text { Realizou um programa de TMAP após a } \\
\text { retirada do cateter (pós-operatório). }\end{array}$ & $\begin{array}{l}\text { A incidência de continência não } \\
\text { apresentou diferença significativa } \\
\text { entre os grupos intervenção e } \\
\text { controle. }\end{array}$ \\
\hline $\begin{array}{l}\text { Hirschhorn } \\
\text { et al., 2014 }\end{array}$ & $\begin{array}{l}139 \\
\text {-Grupo hospital particular ( } \mathrm{n}=107) \\
\text { e hospital público }(\mathrm{n}=32) \text { : Ambos } \\
\text { receberam atendimento sobre o } \\
\text { treinamento da musculatura do } \\
\text { assoalho pélvico durante } 9 \text { meses } \\
\text { antes da intervenção cirúrgica e } 9 \\
\text { meses após. }\end{array}$ & $\begin{array}{l}\text { Intervenção: } \\
\text { Todos os voluntários receberam } \\
\text { treinamento do assoalho pélvico e sobre } \\
\text { sua importância no tratamento através do } \\
\text { enfermeiro ou fisioterapeuta, por material } \\
\text { ilustrativo, DVD e internet. }\end{array}$ & $\begin{array}{l}\text { Houve um aumento significativo } \\
\text { correspondente na prestação de } \\
\text { TMAP pré-operatório por prestadores } \\
\text { do setor privado. Os entrevistados } \\
\text { que receberam TMAP pré-operatório } \\
\text { tiveram melhor autorrelato de IU em } \\
\text { três meses depois da prostatectomia } \\
\text { radical do que aqueles que não } \\
\text { receberam } \\
\text { TMAP pré-operatório. }\end{array}$ \\
\hline Patel et al., 2013 $3^{(20)}$ & $\begin{array}{l}284 \\
\text {-Grupo controle: } \\
\text { Não realizou nenhuma intervenção } \\
\text { supervisionada por um fisioterapeuta } \\
(\mathrm{n}=132) \text {. } \\
\text {-Grupo de intervenção: } \\
\text { Pacientes realizaram exercícios de } \\
\text { Kegel, supervisionados ou orientado } \\
\text { por um fisioterapeuta }(\mathrm{n}=152) \text {. }\end{array}$ & $\begin{array}{l}\text { Grupo controle: } \\
\text { Os pacientes foram instruídos verbalmente } \\
\text { sobre o TMAP pelo cirurgião durante o } \\
\text { período pré-operatório. } \\
\text { Grupo intervenção: } \\
\text { Durante quatro semanas, até a data da } \\
\text { cirurgia, os pacientes realizaram exercícios } \\
\text { de Kegel (10 contrações de } 10 \text { segundos) } \\
\text { sentados, em pé e deitados, sendo } \\
\text { orientados a repeti-los em suas residências. }\end{array}$ & $\begin{array}{l}\text { Após seis semanas de pós-operatório, } \\
\text { o grupo intervenção apresentou } \\
\text { melhora significativa da IU depois da } \\
\text { prostatectomia radical. }\end{array}$ \\
\hline $\begin{array}{l}\text { Marchiori } \\
\text { et al., 2010 }\end{array}$ & $\begin{array}{l}332 \\
\text { - Grupo A: Exercícios para o } \\
\text { assoalho pélvico no pós-operatório, } \\
\text { realizados no local da pesquisa } \\
\text { ( } \mathrm{n}=166) \text {. } \\
\text {-Grupo B: Exercícios de Kegel para } \\
\text { o fortalecimento do assoalho pélvico } \\
\text { no pós-operatório realizado em casa } \\
\text { ( } \mathrm{n}=166 \text { ). }\end{array}$ & $\begin{array}{l}\text { Grupo A: Após } 30 \text { dias da retirada do } \\
\text { cateter, realizou exercícios de Kegel em } \\
\text { sua residência e no local da pesquisa } \\
\text { (em três séries de } 30 \text { contrações diárias, } \\
\text { mantendo a contração alternativamente } \\
1-2 \text { segundos e } 6 \text {-BFB } 7 \text { segundos. BFB } \\
\text { e eletroestimulação com duração de } 30 \\
\text { minutos. } \\
\text { Grupo B: } \\
\text { Realizou exercícios de Kegel em sua } \\
\text { residência durante toda a pesquisa. }\end{array}$ & $\begin{array}{l}\text { A mediana do tempo de recuperação } \\
\text { da continência no Grupo A foi de } 44 \\
\pm 2 \text { dias, enquanto no grupo B foi de } \\
76 \pm 4 \text { dias. Os pacientes do Grupo A } \\
\text { alcançaram continência mais cedo do } \\
\text { que o Grupo B. }\end{array}$ \\
\hline
\end{tabular}




\begin{tabular}{|c|c|c|c|}
\hline $\begin{array}{l}\text { Tienforti et al., } \\
2012^{(22)}\end{array}$ & $\begin{array}{l}32 \\
\text { - Grupo intervenção: Recebeu } \\
\text { sessão de treinamento com BFB e } \\
\text { exercícios de Kegel }(\mathrm{n}=16) . \\
\text {-Grupo controle: Instruções orais e } \\
\text { escritas sobre os exercícios de Kegel } \\
\text { para serem realizados em casa } \\
(\mathrm{n}=16) .\end{array}$ & $\begin{array}{l}\text { Grupo intervenção: } \\
\text { Recebeu uma sessão de treinamento } \\
\text { supervisionado com BFB, instruções orais } \\
\text { e escritas sobre os exercícios de Kegel e } \\
\text { um programa estruturado de exercícios no } \\
\text { pré-operatório. } \\
\text { Grupo controle : } \\
\text { Recebeu, após a remoção do cateter, } \\
\text { apenas instruções orais e escritas sobre os } \\
\text { exercícios de Kegel para serem realizados } \\
\text { em casa. }\end{array}$ & $\begin{array}{l}\text { Os dois grupos foram homogêneos } \\
\text { para todas as características pré e } \\
\text { intraoperatórias analisadas. Porém, } \\
\text { o grupo intervenção apresentou } \\
\text { melhora significativa após um, três } \\
\text { e seis meses quanto à continência } \\
\text { quando comparado ao grupo controle. }\end{array}$ \\
\hline $\begin{array}{l}\text { Rajkowska-Labon } \\
\text { et al., 2014 }\end{array}$ & $\begin{array}{l}81 \\
\text {-Grupo I }(n=49) \text { formou dois } \\
\text { subgrupos }(A I \text { e BI). } \\
\text { AI (n=23): Realizaram BFB, } \\
\text { treinamento do MAP e exercícios } \\
\text { domiciliares. } \\
\text { BI ( } n=26) \text { : Realizaram treinamento } \\
\text { do MAP sem BFB e exercícios } \\
\text { domiciliares. } \\
\text {-Grupo II ( } n=32) \text { : } \\
\text { Não realizou nenhuma intervenção. }\end{array}$ & $\begin{array}{l}\text { Grupo I: } \\
\text { AI: programa de reabilitação em três } \\
\text { partes: a) formação do assoalho pélvico } \\
\text { com BFB, uma vez por semana, duração } \\
\text { de } 20 \text { a } 30 \text { minutos; (b) TMAP de acordo } \\
\text { com a segmentar vertebral, } \\
\text { realizado deitado, sentado e em pé; (c) } \\
\text { exercícios para o paciente realizar por } \\
\text { conta própria em casa ( } 3 \text { vezes por dia, } \\
\text { durante } 15 \text { a } 20 \text { minutos). } \\
\text { B I: outro programa de reabilitação: } \\
\text { (a) TMAP sem BFB, de acordo com a } \\
\text { estabilização da coluna vertebral (duas } \\
\text { vezes por semana, durante } 30 \text { minutos); } \\
\text { (b) exercícios domiciliares idêntico ao do } \\
\text { subgrupo IA. } \\
\text { Grupo II: Não realizou nenhuma } \\
\text { intervenção, sendo acompanhado somente } \\
\text { por ligação telefônica. }\end{array}$ & $\begin{array}{l}\text { Resultados de continência foram } \\
\text { obtidos no Grupo AI x Grupo BI, } \\
\text { e a diferença foi estatisticamente } \\
\text { significativa }(33 / 49 \text { contra } 4 / 32 ; 89 \% \\
\text { versus } 11 \%) \text {. }\end{array}$ \\
\hline $\begin{array}{l}\text { Dijkstra-Eshuis et } \\
\text { al., } 2013^{(24)}\end{array}$ & $\begin{array}{l}122 \\
\text {-Grupo intervenção: Recebeu sessão } \\
\text { de TMAP com BFB. } \\
\text { - Grupo controle: Orientações em } \\
\text { escrito sobre o TMAP. }\end{array}$ & $\begin{array}{l}\text { Grupo intervenção: } \\
\text { Durante quatro semanas antes da } \\
\text { cirurgia, o grupo realizou treinamento } \\
\text { comportamental e BFB, com duração de } \\
30 \text { minutos . } \\
\text { Grupo controle: } \\
\text { Após } 7-10 \text { dias de pós-operatório, os } \\
\text { pacientes receberam orientações em escrito } \\
\text { sobre o TMAP. }\end{array}$ & $\begin{array}{l}\text { O grupo intervenção apresentou } \\
\text { maior taxa de continência em um ano } \\
\text { de seguimento, porém, sem diferença } \\
\text { significante em relação ao grupo } \\
\text { controle. }\end{array}$ \\
\hline $\begin{array}{l}\text { Laurienzo } \\
\text { et al., 2013 }\end{array}$ & $\begin{array}{l}49 \\
\text {-Grupo controle ( } \mathrm{n}=15) \text { : } \\
\text { Não realizou nenhuma intervenção } \\
\text { fisioterapêutica. } \\
\text {-Grupo de exercícios de Kegel } \\
(\mathrm{n}=17) \text { : } \\
\text { Pacientes realizaram exercícios de } \\
\text { Kegel. } \\
\text { Grupo eletroestimulação (ES) } \\
(\mathrm{n}=17) \text { : } \\
\text { Realizou sessões com } \\
\text { eletroestimulação, associadas aos } \\
\text { exercícios de Kegel. }\end{array}$ & $\begin{array}{l}\text { Grupo controle: } \\
\text { Não realizou qualquer tipo de intervenção } \\
\text { terapêutica no período pré-operatório. Os } \\
\text { pacientes desse grupo foram examinados } \\
\text { apenas uma vez, antes da cirurgia, quando } \\
\text { a } \\
\text { avaliação foi realizada e receberam } \\
\text { a informação sobre a anatomia da região } \\
\text { prostática (tal como nos outros grupos). } \\
\text { Grupo exercício de Kegel: } \\
\text { Realizou somente exercícios de Kegel. } \\
\text { Grupo eletroestimulação: } \\
\text { Os pacientes realizaram } 10 \text { sessões, com } \\
\text { estimulação elétrica. Os parâmetros } \\
\text { utilizados foram para fortalecimento } \\
\text { muscular. No pré-operatório, foram } \\
\text { realizados cinco tipos de exercícios de } \\
\text { contração pélvica. }\end{array}$ & $\begin{array}{l}\text { Não foi observada diferença } \\
\text { estatisticamente significante entre os } \\
\text { três grupos de estudo quanto à perda } \\
\text { de urina em pacientes submetidos à } \\
\text { prostatectomia radical retropúbica. }\end{array}$ \\
\hline
\end{tabular}

TMAP = treinamento da musculatura do assoalho pélvico; *IU: incontinência urinária; $\mathrm{BFB}=$ biofeedback; MAP = musculatura pélvica 


\section{RESULTADOS}

Após a aplicação da estratégia de busca na base de dados da Biblioteca Virtual em Saúde, observou-se um total de 26 estudos, sendo 17 da Medical Literature Analysis and Retrieval System Online (MEDLINE), 5 da Central de Registros de Ensaio Clínico Controlado, 2 da Índice Bibliográfico Espanhol em Ciências da Saúde (IBECS) e 2 do Literatura Latino-Americana e do Caribe em Ciências da Saúde (LILACS). Desse total, 17 estudos foram excluídos, devido aos critérios supracitados, culminando em 9 $\operatorname{artigos}^{(17-25)}$.

A idade dos participantes nos estudos variou de 46 a 80 anos, e o tamanho amostral variou de 32 a 120 participantes. Quanto ao tipo de intervenção utilizada, a maior parte dos trabalhos utilizou exercícios para o assoalho pélvico com ou sem biofeedback. O quadro 1 apresenta um resumo dos estudos incluídos nesta revisão.

\section{DISCUSSÃO}

Nove ensaios clínicos controlados foram selecionados para esta revisão, e a análise geral dos estudos combinados sugere que os exercícios para o assoalho pélvico trazem benefícios para o controle da incontinência urinária após a cirurgia de prostatectomia radical.

Um estudo buscou evidenciar se a adição de exercícios do assoalho pélvico nos períodos pré e pós-operatório imediato teria impacto na incidência de continência urinária quando comparado ao uso dos exercícios somente no período pós-operatório. Os resultados apontaram uma incidência de continência urinária maior no grupo que realizou os exercícios nos períodos pré e pós-operatório, porém, sem diferença estatisticamente significante ${ }^{(17)}$.

O mesmo desenho de estudo e os mesmos resultados foram também referidos em outro estudo, com 180 pacientes, ou seja, a adição de exercício no período pré-operatório não apresentou impacto estatisticamente significante na incidência de continência após a cirurgia ${ }^{(18)}$. Uma possível explicação para esse resultado é que os exercícios de curta duração para o assoalho pélvico não são tão eficazes quanto aqueles realizados por um período mais longo.

Ao contrário desses resultados, outra pesquisa, com 139 pacientes de dois hospitais, um público e outro privado, realizou o treinamento do MAP nove meses antes em um grupo e nove meses depois da cirurgia em outro grupo, resultando em uma incidência de contingência urinária significativamente maior quando comparada à do grupo que realizou somente o treinamento do MAP no período pósoperatório ${ }^{(19)}$, resultado similar e que corroborou com outro estudo, com uma amostra de 282 pacientes $^{(20)}$.
A análise desses quatro estudos apontou uma inconsistência quanto à adição dos referidos exercícios no período pré-operatório de prostatectomia radical, no entanto, deve-se salientar que esses resultados devem ser interpretados com cautela, devido à heterogeneidade clínica entre os estudos, especialmente quanto à dosagem dos exercícios no período pré-operatório.

Em um ensaio clínico realizado, os participantes foram divididos em dois grupos (intervenção e controle). O grupo intervenção realizou os exercícios para assoalho pélvico no período pós-operatório, compostos por exercícios de Kegel (fortalecimento da musculatura pubococcígea) associados a biofeedback e eletroestimulação; o grupo controle realizou somente o tratamento clínico convencional. Ao final do estudo, verificou-se que o grupo intervenção alcançou a continência urinária 32 dias mais rápido que o grupo controle $^{(21)}$.

Em estudo semelhante, comparou-se um programa de exercícios de Kegel supervisionado associado à biofeedback e orientações para domicílio no período pré-operatório de cirurgia de PR com um grupo controle que recebeu orientações para domicílio no período pós-operatório. Os resultados apontaram melhora significativa na continência urinária após um, três e seis meses da cirurgia ${ }^{(22)}$.

Divergindo dos três autores supracitados, outra pesquisa analisou a eficácia do treinamento comportamental associado ao biofeedback no período pré-operatório em comparação com um grupo controle que recebeu orientações sobre treinamento da MAP e não encontrou diferença significativa entre os grupos ${ }^{(23)}$.

Os resultados de alguns estudos corroboram ${ }^{(24,25)}$ ao não encontrarem diferença significativa entre dois grupos de tratamentos (um grupo de exercícios de Kegel e outro de eletroestimulação) e um grupo controle que não realizou nenhuma intervenção fisioterapêutica.

Em estudo semelhante aos demais, com 258 pacientes, um trabalho comparou a terapia comportamental para o assoalho pélvico com os exercícios de terapia comportamental associados ao biofeedback e com um terceiro grupo controle que não realizou nenhuma intervenção ${ }^{(26)}$, corroborando com outras literaturas acerca da temática ${ }^{(24,25)}$, apesar de os dois grupos tratamento terem reduzido pela metade os episódios de perda urinária.

A faixa etária nos estudos utilizados mostrou-se muito diversa, porém, já se sabe que o avanço da idade é um fator de risco importante, pois o processo de envelhecimento é um preditor para a manutenção da continência e pode prejudicar a intervenção fisioterapêutica ${ }^{(27)}$.

Considerando que a IU gera estresse e comprometimento da qualidade de vida, qualquer intervenção que possa controlar seus sinais e sintomas deve ser insistentemente 
investigada com o objetivo de diminuir dúvidas e controvérsias sobre suas alternativas de tratamentos. Outro aspecto importante diz respeito ao fato de os exercícios para o assoalho pélvico serem procedimentos não invasivos que podem ter sua utilização como estratégias de prevenção e tratamento da IU após a PR.

\section{CONCLUSÃO}

As evidências científicas apontam que, apesar da heterogeneidade das técnicas e amostras, os exercícios perioperátorios apresentam resultados promissores quanto à minimização da incontinência urinária pós-operatório, principalmente acelerando a recuperação e cura ou diminuindo os sintomas.

\section{REFERÊNCIAS}

1. Kakihara CT, Sens YAS, Ferreira U. Efeito do treinamento funcional do assoalho pélvico associado ou não à eletroestimulação na incontinência urinária após prostatectomia radical. Rev Bras Fisioter. 2007;11(6):481-486.

2. Silva US. Exercícios funcionais do assoalho pélvico no tratamento da incontinência urinária pós prostatectomia radical. Rev Presciência. 2012;10(5):107-18.

3. Billis A. Manual de padronização de laudos histopatológico da Sociedade Brasileira de Patologia. Rio de Janeiro: Reichmann e Affonso; 1999.

4. Prota C. Efeito da reabilitação precoce do assoalho pélvico com biofeedback sobre a disfunção erétil de pacientes submetidos á prostatectomia radical: estudo prospectivo, controlado e randomizado [dissertação]. São Paulo: Faculdade de Medicina da Universidade de São Paulo; 2010.

5. Basiri A, Asl-Zare M, Sischani M, Djaladat $\mathrm{H}$. Laparoscopic bilateral retroperitoneal lymph node dissection in stage II testis cancer. Urol J. 2010;7(3):15760 .

6. Zaindan P, Silva EB. Electrostimulation, response of the pelvic floor muscles, and urinary incontinence in elderly patients post prostatectomy. Fisioter Mov. 2014;27(1):93-100.

7. Bicalho MB, Lopes MHBM. Impacto da incontinência urinária na vida de esposas de homens com incontinência: revisão integrativa. Rev Esc Enferm USP. 2012;46(4):1009-14.

8. Matheus WE, Ferreira U. Incontinência urinária no homem adulto. In: D’Ancona CAL. Princípios básicos de urodinâmica. São Paulo: Atheneu; 1995. p. 65-72.
9. Baracho E. Fisioterapia aplicada á obstetrícia, uroginecologia e aspectos de mastologia. $4^{\mathrm{a}}$ ed. rev. e ampliada. Rio de Janeiro-Guanabara Koogan; 2007.

10. Van Kampen M, De Weerdt W, Van Poppel H, De Ridder D, Feys H, Baert L. Effect of pelvic-floor reeducation on duration and degree of incontinence after radical prostatectomy: a randomized controlled trial. Lancet. 2000;355(9198):98-102.

11. Bauer RM, Bastian PJ, Gozzi C, Stief CG. Postatectomy incontinence: all about diagosis and management. Eur Urol. 2009;55(2):322-33

12. Dubbelman Y, Groen J, Wildhagen M, Rikken B, Bosch R. The recovery of urinary continence after radical retropubic prostatectomy: a randomized trial comparing the effect of physiotherapy ist-guided pelvic floor muscle exercise with guidance by an instruction folder only. BJU Int. 2010;106(4):515-22.

13. Berek JS. Novak: tratado de ginecologia clínica. Rio de Janeiro: Guanabara Koogan; 2005.

14. Kakihara, CT; Ferreira, U; Pedro, RN; Matheus, WE; Junior, NRN. Archivos Españoles de Urologia, Campinas, vol. 59, n. 8, p. 773-778, 2006.

15. Berghmans B. El papel del fisioterapeuta pélvico. Actas Urol Esp. 2006;30(2):110-22.

16. Lima SVS. Fisioterapia: A relevância no tratamento da incontinência urinária. Rev Eletrônica Novo Enfoque. 2010;10(10):144-60

17. Centemero A, Rigatti L, Giraudo D, Lazzeri M, Lughezzani G, Zugna D, et al. Preoperative pelvic floor muscle exercise for early continence after radical prostatectomy: a randomised controlled study. Eur Urol. 2010;57(6):1039-43.

18. Geraets S, Poppel HV, Devoogdt N, Joniau S, Van Cleynenbreugel B, Groefa N et al. M. Influence of Preoperative and Postoperative Pelvic Floor Muscle Training (PFMT) compared with Postoperative PFMT on Urinary Incontinence After Radical Prostatectomy: a randomized controlled Trial. Eur Urol. 2013;64(5): 766-72.

19. Hirschhorn AD, Kolt GS, Broks AJ. A multicomponent theory based intervention improves uptake of pelvic floor muscle training before radical prostatectomy: a before and after cohort study. BJU Int. 2014;113(3): 383-92.

20. Patel MI, Yao J, Andrew D, Mungovans SF. Preoperative pelvic floor physiotherapy improves continence after radical retropubic prostatectomy. Int J Urol. 2013;20(10):986-92 
21. Marchiori D, Bertaccini A, Manferrari F, Martorana G. Pelvic floor rehabilitation for continence recovery after radical prostatectomy: role of a personal training reeducational program. Anticancer Res. 2010;30(2):5536.

22. Tienfortid SE, Marangi F, D'addessi A, Racioppi M, Giulino G, Pinto F et al. Efficacy of an assisted low-intensity programme of perioperative pelvic floor muscle training in improving the recovery of continence after radical prostatectomy: a randomized controlled trial. BJU Int. 2012;110(7):1004-10.

23. Rajkowska-Labonei H, Kucharzewski M, Bigniewu L. Efficacy of physiotherapy for urinary incontinence following prostate cancer surgery. Bio Med Research International. 2014:785263.

24. Dijkstra-Eshuis J, Van den Bos TW, Splinter R, Bevers RF, Zonneveld WC, Putter H. Effect of preoperative pelvic floor muscle therapy with biofeedback versus standard care on stress urinary incontinence and quality of life in men undergoing laparoscopic radical prostatectomy: a randomised control trial. Neurourol Urodyn. 2015;34(2):144-50

25. Laurienzo CE, Sacomomani CA, Rodrigues TR, Zequi SC, Guimarães GC, Lopes A. Results of preoperative electrical stimulation of pelvic floor muscles in the continence status following radical retropubic prostatectomy. Int Braz J Urol. 2013;39(2):182-8.
26. Goode PS, Burgio KL, Johnson TM, Clay OJ, Roth DL, Markland AD. Behavioral therapy with or without biofeedback and pelvic floor electrical stimulation for persistent postprostatectomy incontinence a randomized controlled trial. JAMA. 2011;305(2): 151-9.

27. Castro AP, Pereira VS, Serrão PRMS, Driusso P. Eficácia do bioffedback para o tratamento de incontinência urinária por esforço: uma revisão sistemática. Sci Med. 2010;20(3):257-63.

\section{Endereço do primeiro autor:}

Adonivia Guimarães Santos

Faculdade Inspirar

Rua Inácio Lustosa, 792

Bairro: São Francisco

CEP 80510-000 - Curitiba - PR - Brasil

E-mail: nivea.guimaraes@hotmail.com

\section{Endereço para correspondência:}

\section{Luisa Braga Jorge}

Pontifícia Universidade Católica - PUC - Porto Alegre Av. Ipiranga, 6681

Bairro: Partenon

90619-900 - Porto Alegre - RS - Brasil

E-mail: lbragajorge@hotmail.com 